\title{
Structural Monitoring System for proactive detection of corrosion and coating failure
}

\author{
Jawwad Latif ${ }^{1}$, Zulfiqar A Khan* ${ }^{1}$, Keith Stokes ${ }^{2}$ \\ ${ }^{1}$ Bournemouth University, Department of Design and Technology, NanoCorr Energy and Modelling \\ (NCEM) Research Group \\ ${ }^{2}$ University of Southampton, National Centre for Advanced Tribology at Southampton (nCATS) \\ "Corresponding author email: zkhan@bournemouth.ac.uk
}

\begin{abstract}
The performance and availability of high priority structures can be greatly affected by corrosion damage. The application of protective coatings, frequent inspections and scheduled based maintenance activities result in huge direct and indirect financial loss to organisations. The expeditious detection of coating failure and corrosion damage can result in precise and costeffective condition-based maintenance. Coating failure and corrosion phenomena are driven by complex multi-disciplinary parameters according to extensive research findings in the literature. State-of-the-art prognostic models proposed in recent years incorporate complex multi-disciplinary parameters, therefore a real-time prognostic monitoring system must acquire these complex parameters to allow accurate prediction. The work reported here covers the development of a realtime monitoring system using micro-sensors and includes the validation of the system through accelerated corrosion and coating failure testing. The system contains a remote terminal unit that includes a linear polarisation method for corrosion detection under the coating and a micro-strain gauge method for monitoring stress behaviour over the coating. The software at base station includes a graphical user interface and database to store parameters for further processing and failure prediction. The real-time monitoring system can be applied to remote, stationary and mobile assets to monitor the mechanical and chemical changes within coating-substrate systems.
\end{abstract}

\section{Introduction}

Critical structures including aircraft, automobiles, highway bridges, energy production, distribution systems, and household appliances can be highly susceptible to corrosion damage due to excessive operational activities in uncontrolled environmental conditions. According to recent studies, the direct cost of corrosion damage is around 3\% of the national Gross Domestic Product [1]. Indirect costs, including the cost of operator activities, the overhead cost of delays, loss in productivity and any litigation issues are estimated as being equally costly.

Durable organic coatings are applied to structures to provide a protective barrier from surrounding environmental conditions. The organic coatings are susceptible to numerous types of failure modes including micro-cracking within the coating surface which allows the corrosive species to diffuse into the coating-substrate interface to initiate corrosion damage [2]. According to recent experimental investigations, parameters including interfacial roughness, interfacial toughness, diffusion-induced stresses and residual stresses are considered to be responsible for micro-cracks and adhesion loss in protective coatings applied to metal structures [2-12]. Various maintenance activities are implemented to protect and repair the metal structures e.g. brushing, washing and recoating. The 
maintenance of critical metal structures, therefore, can be costly and time-consuming due to carrying out excessive inspection and recoating maintenance, coupled with any delay in productivity. The most commonly practiced scheme for maintenance is referred to as Scheduled Based Maintenance (SBM) which is carried out at regular intervals independent of the condition of the structure. This often results in unnecessary maintenance and has led to studies into alternative, more cost effective, approaches over the past decade. These studies have resulted in the implementation of Condition Based Maintenance (CBM), where the condition of the structure indicates that maintenance is necessary. The approach to identify the need to implement such maintenance strategies ideally needs to be low cost, simple to install and operate. Sensor technology can provide an efficient solution for condition monitoring of structures operating at remote locations.

The corrosion mechanism lies under electrochemistry concepts while residual stresses are mechanistic parameters. To address the former, various corrosion sensors have been developed and are characterised as direct and indirect corrosion monitoring techniques. The direct monitoring technique involves the measurement of potential or current resulting from corrosion/electrochemical reactions. Corrosion coupons, electrochemical impedance spectroscopy, electrical resistance and potential measurement using linear polarisation are examples of direct corrosion monitoring techniques. These techniques are also termed as intrusive as they require access to the metal structure for corrosion measurement. Indirect corrosion monitoring techniques are based on an outcome resulting from corrosion reaction. Radiography and ultrasonic testing are common indirect and non-intrusive corrosion monitoring techniques. Both techniques have advantages and disadvantages. The corrosion rate from corrosion coupons is estimated by measuring the weight-loss over a particular length of time. It provides a reliable physical indication of the overall uniform corrosion rate but is time-consuming and cannot be used in real-time monitoring systems [13]. The electrical resistance method measures the change in resistance caused by metal loss. The rate of corrosion can be measured at any time and as frequently as needed but requires calibration based on the properties of the structural material [14]. Electrochemical Impedance Spectroscopy is used to measure the corrosion rate of the metal structure as well as any corrosion occurring under the coating. It is a powerful method of determining corrosion rate, but it is very sophisticated and prone to noise in the field environment. Linear Polarisation Resistance (LPR) method is also a powerful tool that provides a direct and instantaneous determination of corrosion rate which can be utilised for real-time structural monitoring. The disadvantage of using LPR technique, however, is that it is only effective in clean, aqueous electrolytes.

The performance of $\mu$ LPR sensors has been evaluated using an accelerated corrosion test to ASTM G5-A [13]. From these tests it was established that $\mu L P R$ sensors can precisely measure the low corrosion rate in corrosive atmospheric conditions. Using these sensors, the NanoCorr Energy Modelling and Research (NCEM) group have investigated corrosion damage within large vehicles at The Tank Museum, Bovington, Dorset UK. The $\mu$ LPR sensors were embedded on vehicles operating in both controlled and uncontrolled environment at remote locations [4, 15]. The Tank Museum is located near the English Channel, hence a high amount of salinity is present in the atmosphere. The average wind speed always remains greater than the threshold level for entrainment of salt particles. The relative humidity level also remains greater than the critical threshold level throughout the calendar year. The valuable vehicles operating in these harsh atmospheric conditions are highly prone to corrosion damage. The temperature and humidity levels inside the museum are controlled 
and kept at appropriate levels to provide suitable environmental conditions to large vehicles. While the harsh atmospheric conditions outside of the museum is considered as uncontrolled environmental conditions. The $\mu \mathrm{LPR}$ sensors embedded on the stationary vehicles in the controlled environment have not indicated any significant signs of corrosion, while those embedded on the stationary vehicles at remote locations in the uncontrolled environment have detected corrosion reactions.

The residual stresses within the coating-substrate system are one of the major factors for coating failure in the form of blistering and micro-crack opening as shown in Figure 1. The stresses could be tensile and compressive depending on material properties and temperature gradient. According to past experimental study, the coefficient of thermal expansion (CTE) property of coating and substrate is responsible for the occurrence of tensile and compressive stresses, depending on change in temperature [2]. The tensile stress opens the cracks on the coating and allows corrosive particles to diffuse into the coating-substrate interface, subsequently resulting in corrosion under the coating as shown in Figure 1. The compressive stresses control the number of corrosive particles which diffuse through the coating due to contraction and they can also generate blisters if they exceed a critical level [6]. Several methods are applied to measure residual stresses which are characterised as Non-destructive, Semi destructive and Destructive techniques [16]. X-ray diffraction, Neutron diffraction, Ultrasonic and Barkhausen noise methods lie within the Nondestructive category. Hole-drilling, Ring core and Deep-hole methods are semi-destructive techniques while Sectioning and Contour methods are destructive techniques used for residual stress measurement.

The behaviour of tensile and compressive stresses in coatings needs to be monitored in real time for efficient prediction of potential structural damage. Although strain gauge sensors have been widely used in aerospace applications, bridge cables, measurement of torque generated by motors, turbines, propellers or wheels, the performance of strain gauges in monitoring corrosion and coating damage assessment needs to be investigated in more detail. The residual stresses generated during physical vapour deposition coating have been scaled to real-time using the strain gauge sensor. The experimental data generated from strain gauge monitoring has been validated with predicted values of residual stress. The performance of strain gauges in monitoring the development of residual stresses in the coating-substrate system due to change in temperature has also been investigated [17]. In the current work, strain gauge sensors have been used to acquire real-time measurements of residual stresses developing in the coating. The proposed structural monitoring system is based on a sensor suite to detect electrochemical reactions using $\mu$ LPR sensors and mechanical residual stresses using strain gauge sensors. In addition, temperature and humidity sensors are included within the suite. 

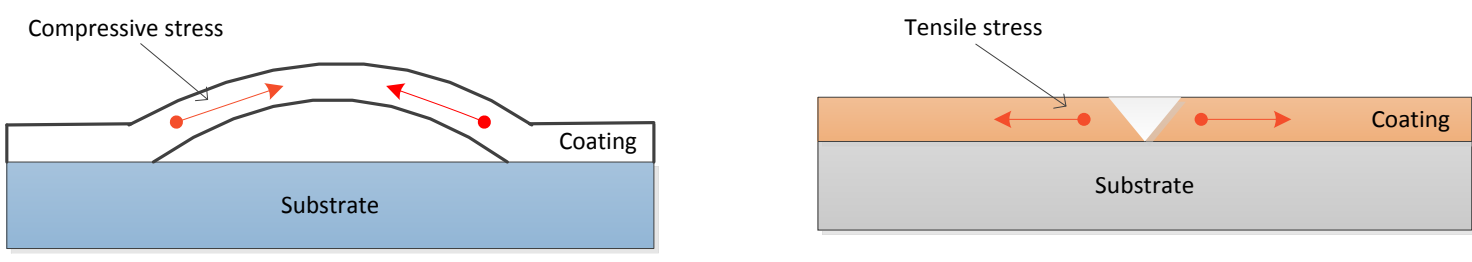

(a) Compressive stress in coating

(b) Tensile stress in coating

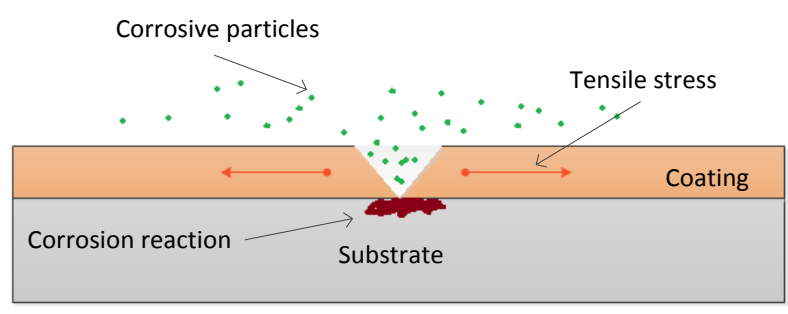

(c) Corrosion under the coating

Figure 1 Coating failure and corrosion phenomena

\section{Proposed Structural Monitoring System}

The Tank Museum, Bovington, Dorset is located near the English Channel in Dorset where large military vehicles are exhibited and operated for various purposes. The presence of high amounts of salt particles in the atmosphere combined with wind speed, rain, relative humidity and temperature are causing a potential threat to their structural integrity. It has been established in the literature that the stresses within a coating control the compression and opening of micro-cracks that allows corrosive salt particles to diffuse into the interface of coating and substrate [2,10]. The proposed system monitors the mechanical and chemical changes through $\mu$-strain gauge and $\mu$ LPR sensors. The $\mu$-strain gauge sensors are used to monitor the behaviour of stresses over the coating due to change in temperature, whilst the $\mu L P R$ sensors are used beneath the coating to monitor any corrosion reactions that occur due to the diffusion of salt particles through micro-cracks. The proposed structural monitoring system is shown in Figure 2.

Consider a coating-substrate system where the applied coating has a higher value of coefficient of thermal expansion than the substrate. In past studies, It was observed that when the temperature drops, the coating tries to shrink but the substrate, having the lower value of coefficient of thermal expansion (CTE) restricts contraction of the coating. As a result the coating experiences tensile stress which opens the crack and allows corrosive particles to diffuse through [2], as shown in Figure 2. As stated, the current sensor suite contains temperature, strain and $\mu L P R$ sensors, where the temperature sensor monitors change in temperature, the strain gauge monitors the development of residual stresses and the $\mu \mathrm{LPR}$ sensor detects any corrosion reaction. The complete structural monitoring system consists of a terminal unit with various sensors and software at a remote base station. 


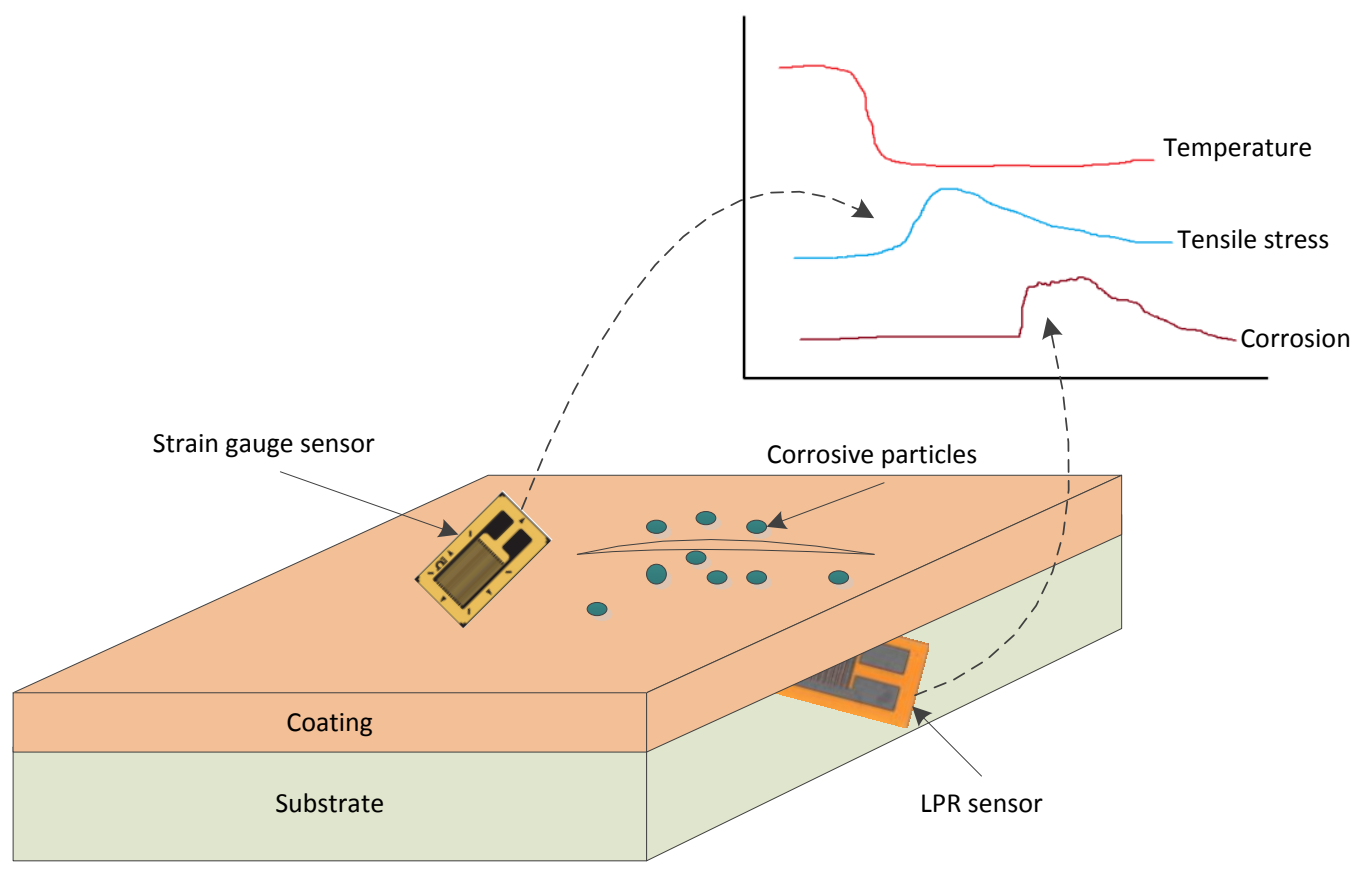

Figure 2 Proposed structural monitoring system

\subsection{Remote Terminal Unit}

Large metal structures are comprised of large and complex geometrical configurations, therefore various types of sensors are required at critical locations on the structure. Arduino Mega 2560 Rev3 is used to develop the remote terminal unit (RTU). The Arduino boards are an open source and programmable electronic circuit board which consists of microcontroller. It can be programmed using Integrated Development Environment (IDE) software to design customise embedded systems for various applications. The RTU has various channels for corrosion, strain gauge, temperature and humidity sensors. The architecture of RTU is described through the flow chart in Figure 3.

The RTU must be synchronised with the current time and date. The interval between data sampling is adjusted depending on the sensitivity and environmental conditions of the structure. The external Real Time Clock (RTC) is embedded within the Arduino Mega 2560 Rev3 and adjusts the time interval for activating the sensors for measuring and transmitting data to the base station. The Global System for Mobile communications (GSM) is used for wireless transmission and allows both stationary and mobile structures to be monitored. 


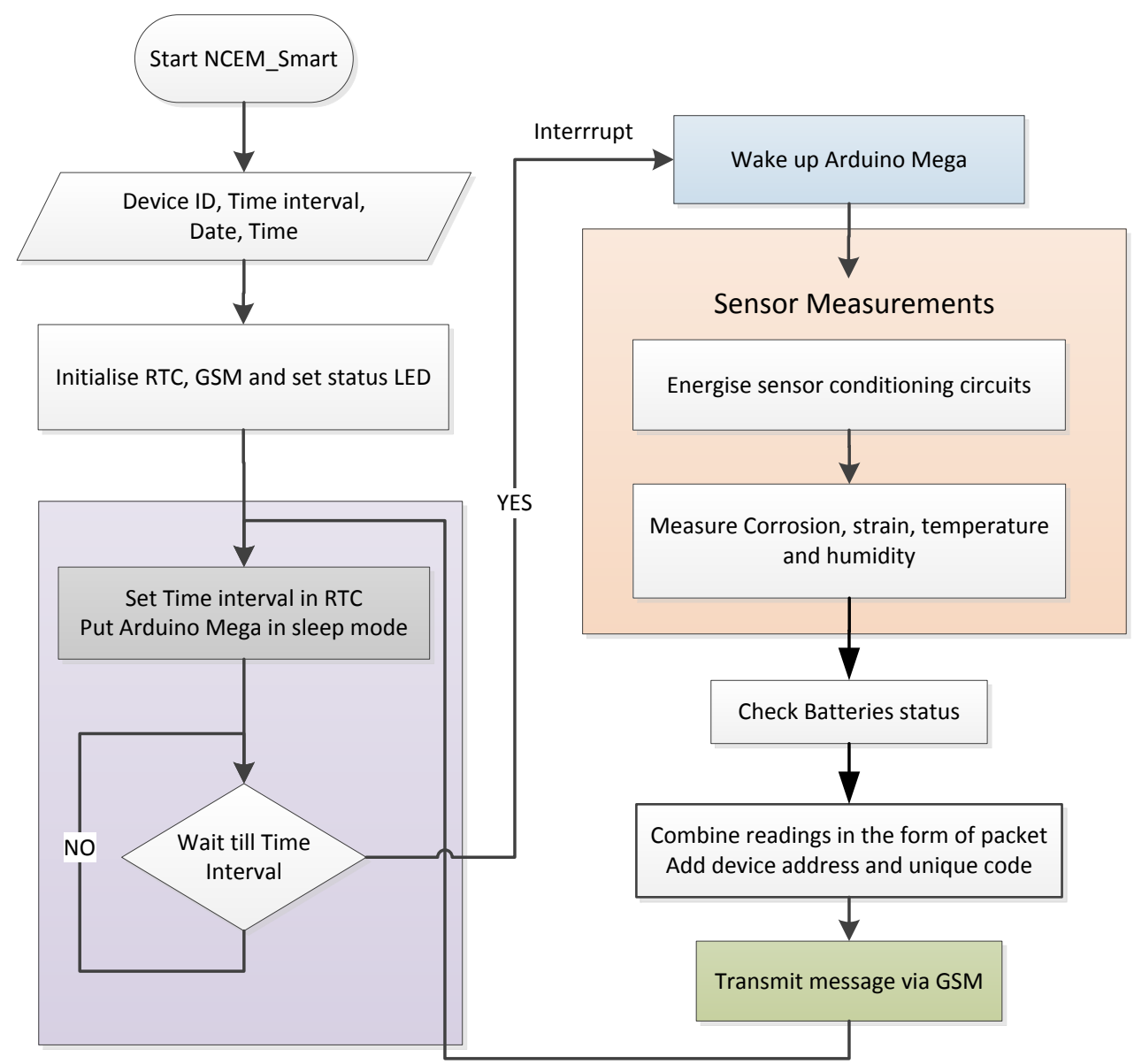

Figure 3 Flowchart for RTU

\subsubsection{Corrosion monitoring technique}

In this current work, the Linear Polarisation Resistance method is used for detecting the corrosion reaction at the interface between coating and substrate. Corrosion is an electrochemical process which occurs due to the oxidation (anodic) reaction, which involves loss of metal electrons, and the reduction (cathodic) reaction, which involves gain of electrons, in the presence of an electrolyte such as oxygen and water [18]. The oxidation and reduction reactions occur at random locations on the metal surface in the presence of aqueous solution. The oxidation site on the metal results in the loss of an electron(s) which can be written as:

$$
M_{(s)} \longrightarrow M_{(a q)}^{n+}+n e^{-}
$$

where ' $M$ ' represents the metal, which is losing ' $n$ ' number of electrons. The chemical equation for the cathodic reaction in the presence of an aqueous solution gaining electrons can be written as:

$$
\mathrm{n} \mathrm{H}_{z} \mathrm{O}+\mathrm{n} e^{-} \longrightarrow \mathrm{nOH} H^{-}+(\mathrm{n} / 2) \mathrm{H}_{z}
$$

The corrosion reaction occurring on steel in the presence of water is shown in the Figure 4. 


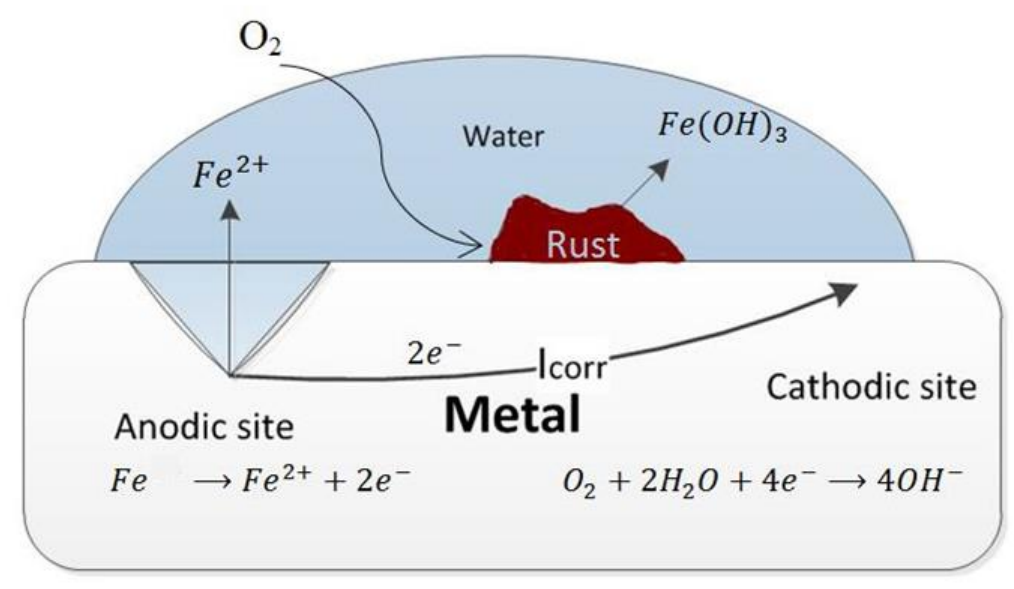

Figure 4 Corrosion reactions

The $\mu$ LPR sensor is based on three electrode system theory and includes working, counter/auxiliary and reference electrodes. The working electrode is in electrical contact with the structure to be monitored and acts as the anode. The counter electrode completes the circuit and acts as the cathode. The reference electrode has a stable known potential. The basic architecture of the $\mu$ LPR is shown in Figure 4. The $\mu$ LPR sensor developed by ANALATOM as shown in Figure 5 has been utilised for corrosion detection in the current work and has been previously discussed [13].

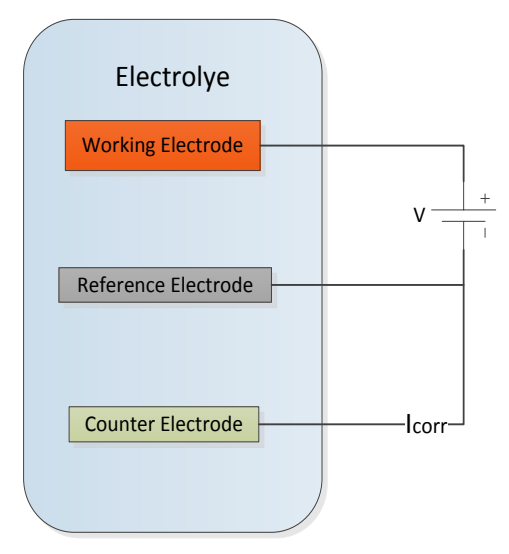

Figure 4 Three electrode system

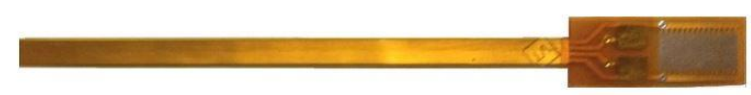

(a)

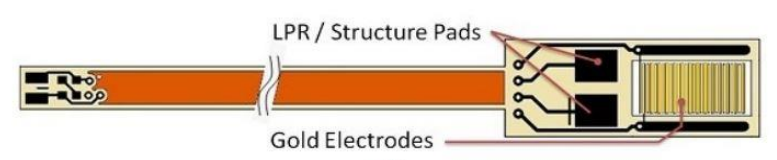

(b)

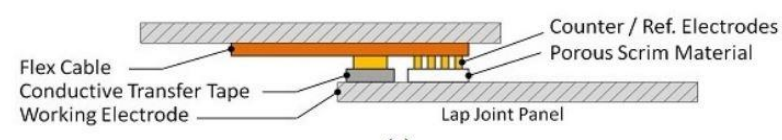

(c)

Figure 5 Structure of $\mu$ LPR sensor [13]

Once the value of polarisation resistance is measured through the $\mu$ LPR sensor, the Stern-Geary equation is applied to calculate the corrosion rate as follows [15]:

$$
I_{\text {Corr }}=\frac{\mathrm{B}}{\mathrm{R}_{\mathrm{p}}}
$$

'I $I_{\text {Corr }}$ ' represents the corrosion current, ' $\mathrm{R}_{\mathrm{p}}$ ' is polarisation resistance and ' $\mathrm{B}$ ' is a proportionality constant which depends on anodic and cathodic Tafel slopes. The relation for proportionality constant ' $B$ ' is given as: 


$$
B=\frac{\beta_{a} \beta_{c}}{2.303\left(\beta_{a}+\beta_{c}\right)}
$$

In the above equation, ' $\beta_{a}$ ' is the anodic Tafel slope and ' $\beta_{c}$ ' is the cathodic Tafel slope. The Tafel slopes can be plotted and measured using ASTM standard G59 [19]. The corrosion rate is then determined using the following equation:

$$
\text { Corr_Rate }=\mathrm{I}_{\text {Corr }}\left[\frac{w}{A * e * F}\right]=\frac{\mathrm{B}}{\mathrm{R}_{\mathrm{p}}}\left[\frac{w}{A * e * F}\right]
$$

The ' $w$ ' represents atomic weight, ' $F$ ' represents Faraday's constant, ' $e$ ' is the number of electrons transferred during the corrosion process and ' $A$ ' is the area of the corroding electrode.

Figure 6 shows the electronic circuit for a $\mu$ LPR sensor that is connected to the Arduino Mega. Corrosion occurring on the sensor causes a change in resistance which then results in a change in voltage. As the magnitude of voltage is very low, it requires amplification and this is achieved using the IC-741. The gain can be calculated using the following relationship:

$$
G=1+\frac{R_{f}}{R}
$$

Where the value of $R_{f}=100 k$ and $R=10 k$, respectively. The measured voltage is converted into corrosion current using mathematical relations programmed in the Arduino Mega.

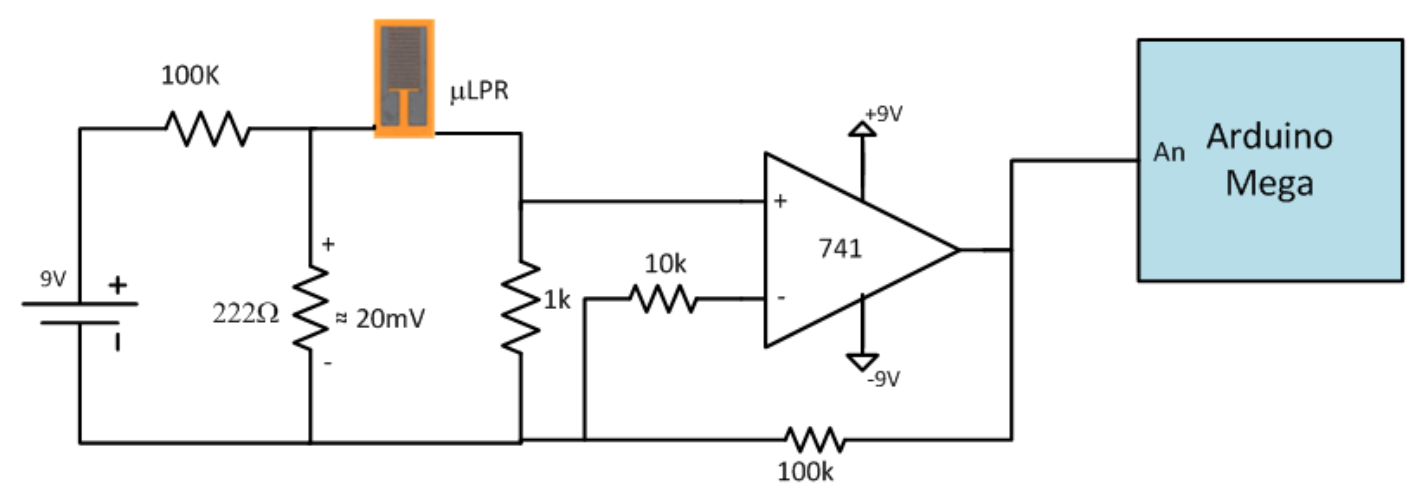

Figure 6 Electronic circuit for $\mu$ LPR sensor

\subsubsection{Stress monitoring technique}

Stresses in coatings are primarily due to poor coating application and the temperature gradient. The value of the coefficient of thermal expansion of coating and substrate defines the magnitude of stress and strain. The stresses result in deformation of the coating in the form of contraction and widening of micro-cracks. The strain gauge sensor can be used to monitor the strain produced in the coating due to change in temperature and consists of a foil pattern which deforms as the coating deforms due to stresses. It is embedded on the structure or sample with an adhesive bond and its resistance changes as it deforms. The change in resistance is measured by connecting the strain 
gauge into a Wheatstone bridge which is used to determine the unknown resistance in the electrical circuit. The circuit diagram of the strain gauge connected into the Wheatstone bridge is shown in Figure. 7. The data acquisition unit contains a Wheatstone bridge and a strain gauge is connected in a quarter bridge configuration.

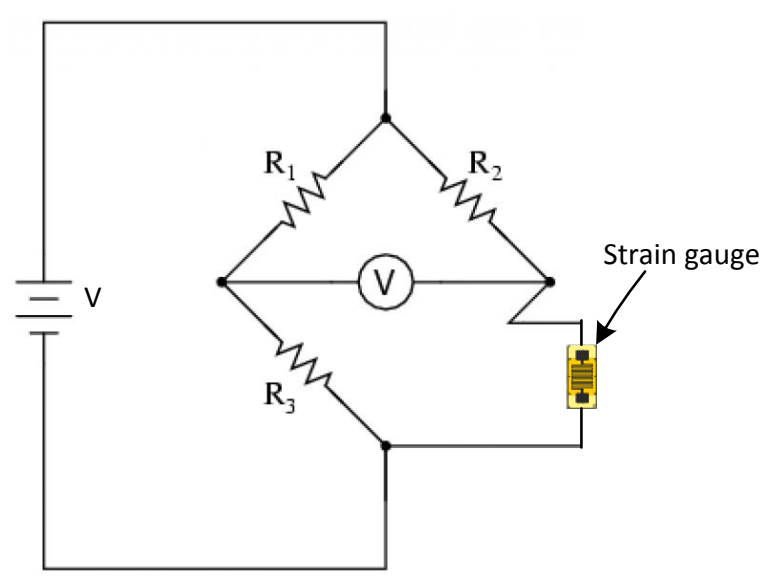

Figure 7 Wheatstone bridge with strain gauge sensor

Figure 8 show the electronic circuit for a $\mu$-strain gauge sensor that is connected to the Arduino Mega. The change in strain causes a change in resistance, which then results in a change in voltage level. The magnitude of the current is very low; therefore it is amplified using the IC-741. The $\mu$ strain gauge sensor is connected as a resistance within the Wheatstone bridge and it follows the following relationship:

$$
\frac{R_{1}}{R_{2}}=\frac{R_{0}}{R_{x}}=1
$$

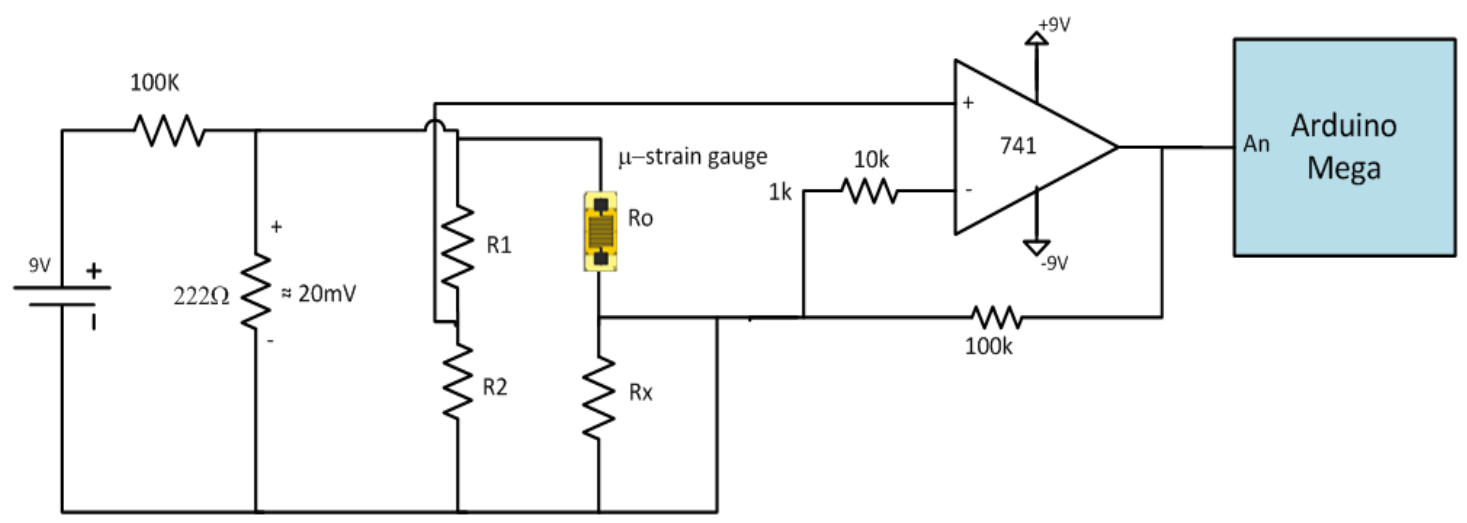

Figure 8 Electronic circuit for $\mu$-strain gauge sensor

The DHT22 sensor as shown in Figure 9 is used for temperature and humidity measurements. The RTU also monitors battery life using the electronic circuit shown in Figure 10. 


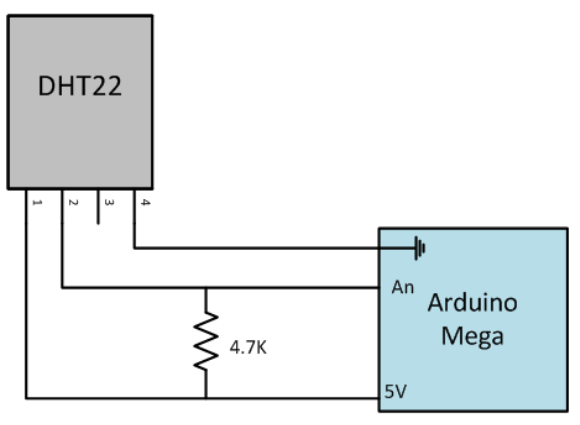

Figure 9 Electronic circuit for DHT22 sensor

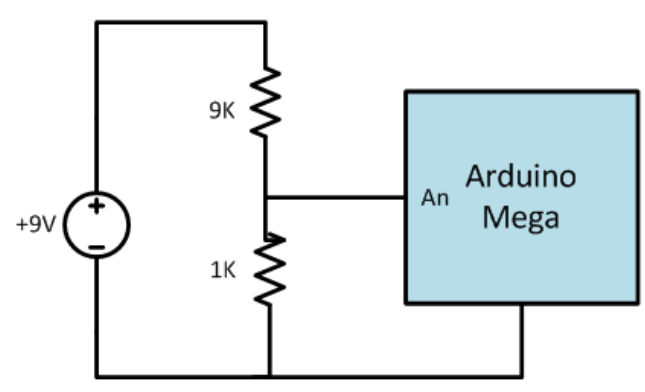

Figure 10 Electronic circuit for battery life

The RTU transmits the data packet to the remote base station with all required information as shown in Figure 11. A unique code acts as an address for the device and validation of the message from the RTU. It also contains date and time stamps associated with the sensor measurements. The complete RTU system is shown in Figure 12.

\section{Data Packet}

\begin{tabular}{|c|c|c|c|c|c|}
\hline Unique Code & Date & Time & Sensor Values ......N & $\begin{array}{c}\text { Battery } \\
\text { status/Fault }\end{array}$ & Unique Code \\
\hline
\end{tabular}

Figure 11 Data packet contains all information

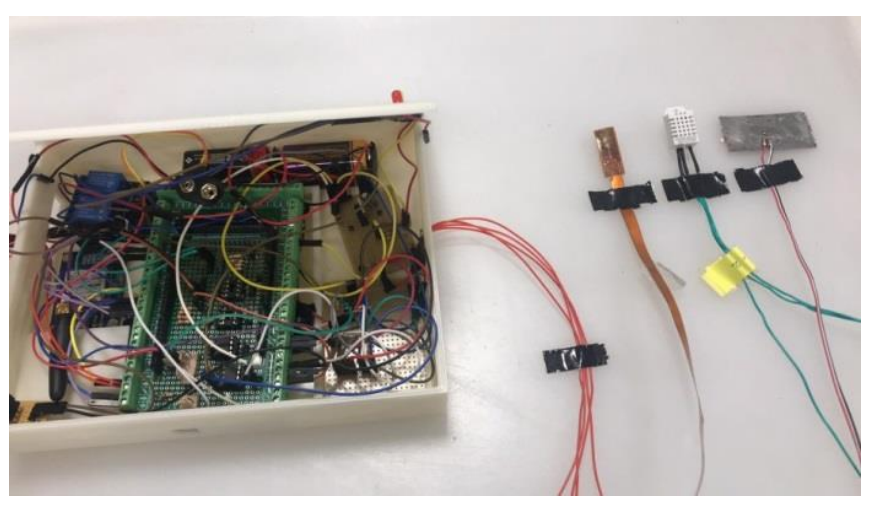

(a) Internal view

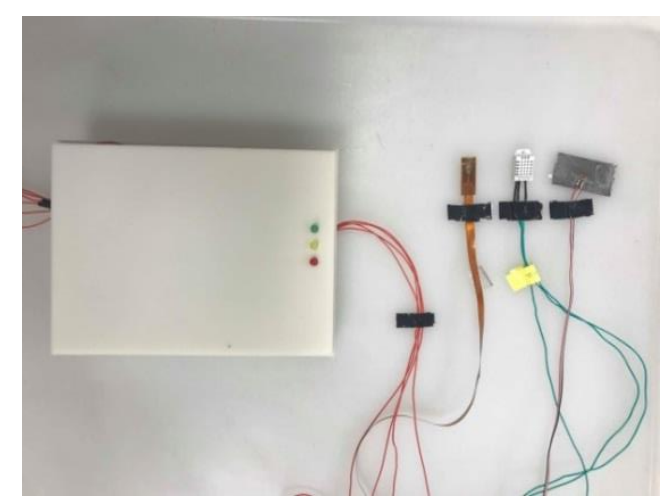

(b) External view

Figure 12 RTU system with sensors

\subsection{Base Station}

The software at the base station is configured to the GSM module in order to communicate to the RTU's. The flow chart shown in Figure 13 demonstrates how the base station receives a data packet from the RTU and processes them for further analysis. The information is extracted from the data packet one by one and operations are performed accordingly. It starts by identifying the structure 
using a unique address of RTU. The sensor values associated with time and date are extracted and stored in the corresponding database of the structure. The threshold for any critical value can also be defined to show live critical status on the Graphical User Interface. The complete system at the base station which contains software and GSM is shown in Figure 14.

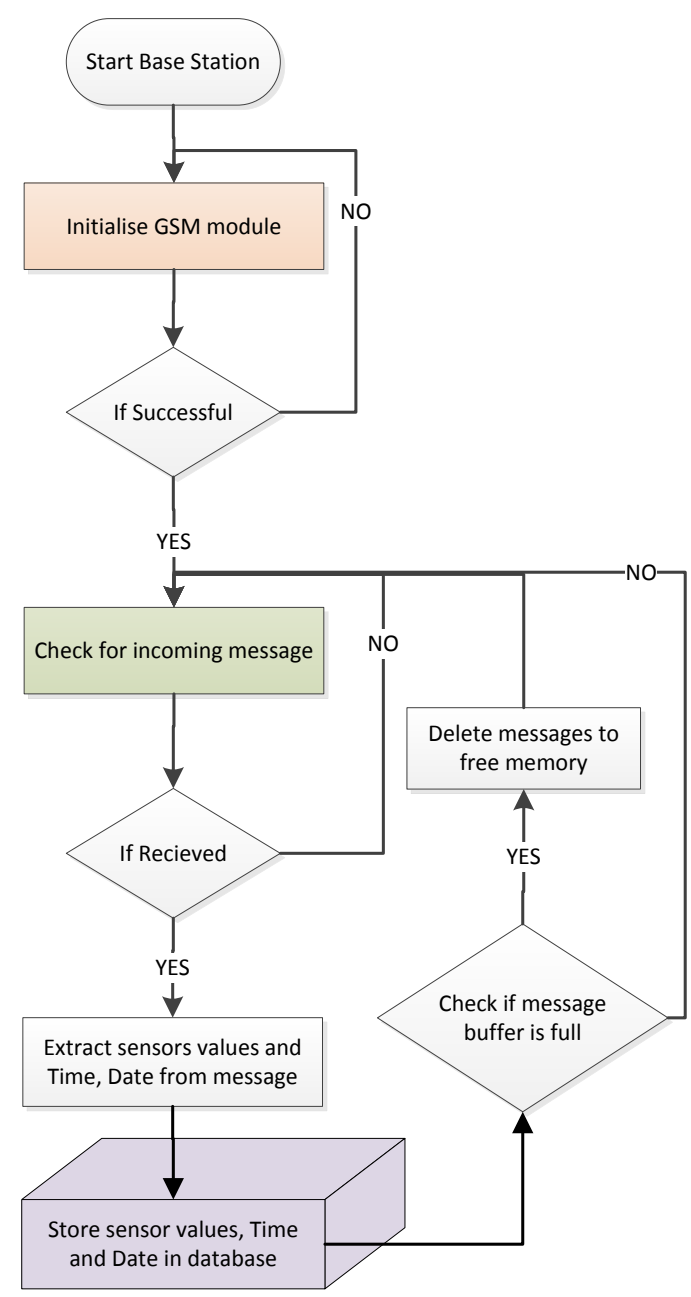

Figure 13 Flow chart for Base Station 


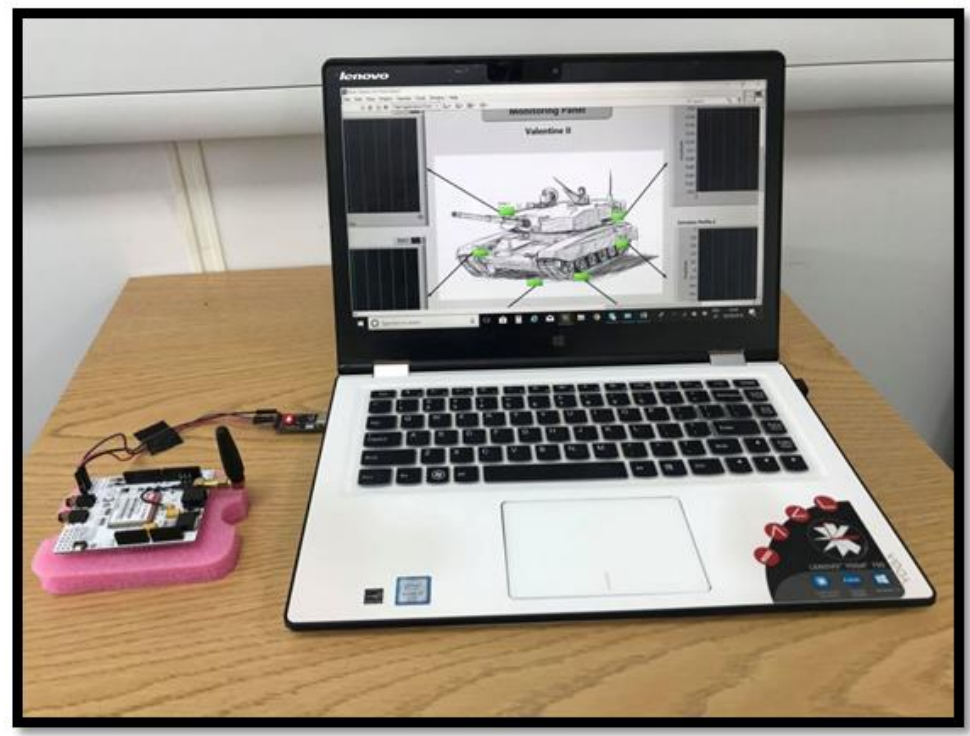

Figure 14 Base station system

In order to verify the wireless communication and response of the sensors, the system was repeatedly tested in salt solution and an accelerated corrosion environment inside the laboratory as shown in Figures 15 and 16. The salt spray chamber provided an accelerated corrosion environment using salt solution. The sensors were kept inside the chamber and the response of these sensors and the wireless communication was monitored at the base station.

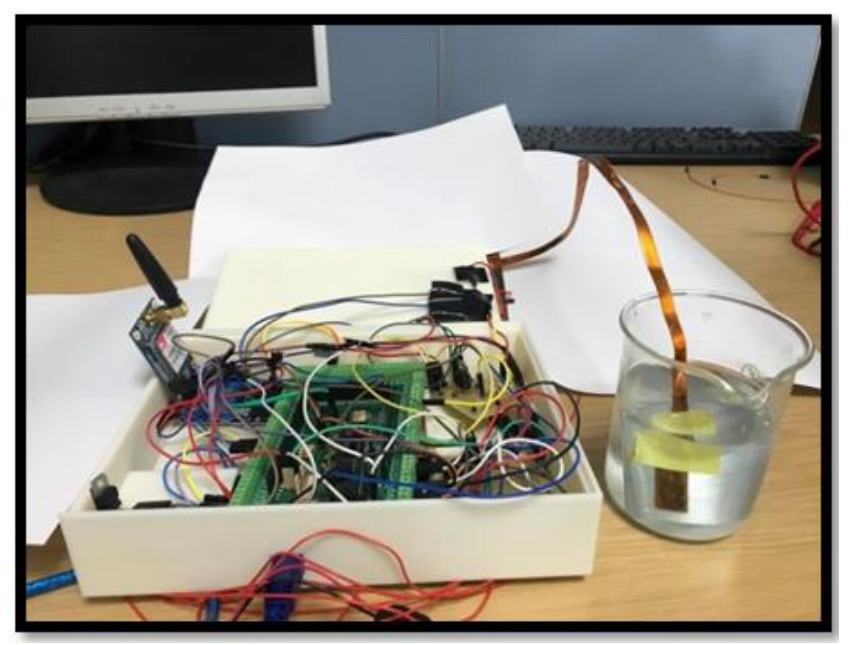

Figure 15 Inserting a corrosion sensor in salt solution to validate the corrosion detection 


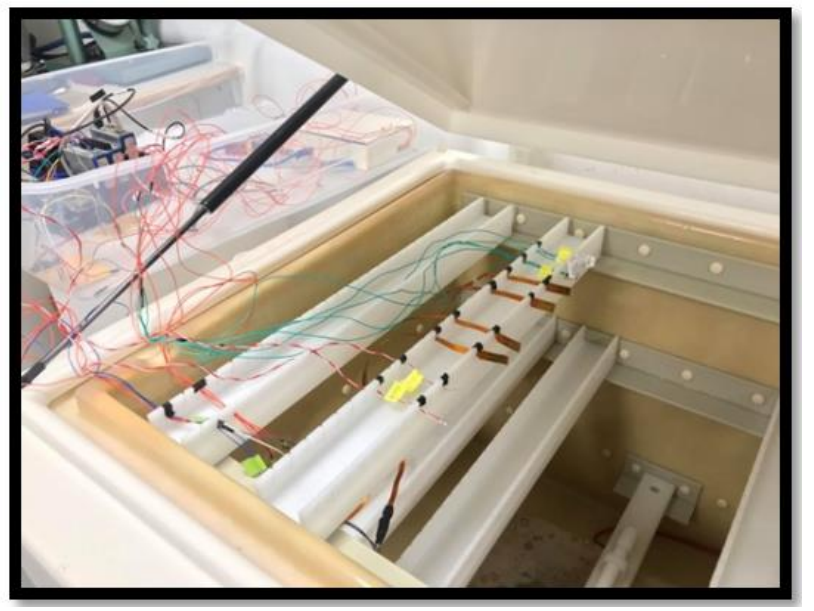

Figure 16 Sensors inside the salt spray chamber

\section{Experimental setup}

The proposed sensor-based system was validated through accelerated corrosion testing on metal samples coated with primer. Three samples of aluminum 6082 with dimensions $50 \mathrm{~mm} \times 50 \mathrm{~mm}$ and thickness $3 \mathrm{~mm}$ were prepared. The coefficient of thermal expansion of aluminum 6082 is $24 \times 10^{-6} \mathrm{~K}^{-1}$ and Modulus of Elasticity is $70 \mathrm{GPa}$ [20]. Sample preparation involved the following steps:

- Sample polishing

- Installation of the $\mu$ LPR sensor on the metal substrate (prior to coating

- Application of primer red-oxide coating on samples

- Installation of strain gauge sensor on coated sample

The interfaces of the aluminum 6082 samples were polished with an emery paper of 80 grit size and are shown in Figure 17. The samples were washed with deionised water following polishing. The roughness of the interfaces after polishing was measured at 8 different points using Optical Inferometery. The average roughness of each sample is given in the Table 1.

Sample 1

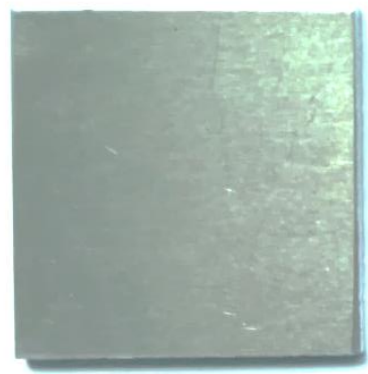

Sample 2

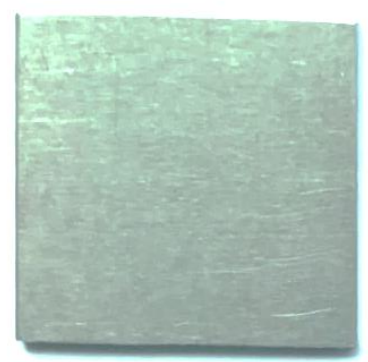

Figure 17 Samples after polishing
Sample 3

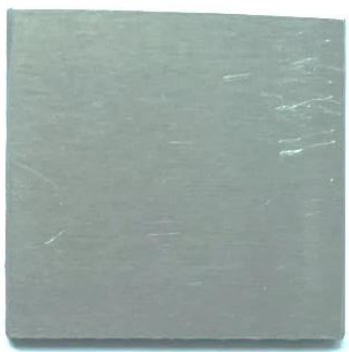


Table 1 Surface roughness of each sample

\begin{tabular}{|l|c|c|c|}
\hline & $\begin{array}{c}\text { Sample 1 } \\
\operatorname{Ra}(\mu \mathrm{m})\end{array}$ & $\begin{array}{c}\text { Sample 2 } \\
\operatorname{Ra}(\mu \mathrm{m})\end{array}$ & $\begin{array}{c}\text { Sample 3 } \\
\operatorname{Ra}(\mu \mathrm{m})\end{array}$ \\
\hline Average Roughness & 1.501 & 1.197 & 1.335 \\
\hline
\end{tabular}

The $\mu \mathrm{LPR}$ sensor, with dimensions $40 \mathrm{~mm} \times 20 \mathrm{~mm} \times 0.1 \mathrm{~mm}$, was then attached to the sample interface ensuring electrical contact to the working electrode of $\mu$ LPR sensor. Adhesive tape was used to keep the $\mu \mathrm{LPR}$ sensor in place before the coating is applied, as shown in Figure 18. The $\mu \mathrm{LPR}$ sensor is connected to the RTU which converts the electrical signals into digital signals and forwards the data to the base station.

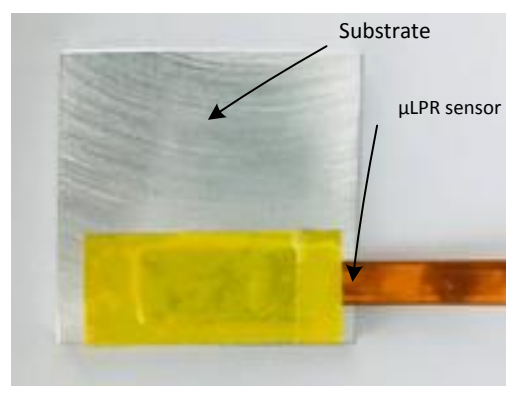

Figure 18 Sample with $\mu$ LPR sensor

The red oxide primer, with a coefficient of thermal expansion of $21 \times 10^{-6} \mathrm{~K}^{-1}$ and a Modulus of Elasticity of $6.14 \mathrm{GPa}$, was applied on the sample with a conventional spray gun at a temperature of $300 \mathrm{~K}$. The coated sample was allowed to dry for $24 \mathrm{hrs}$ before the next coating was applied. The red oxide primer was applied three times and completely covered the sample as shown in Figure 19.

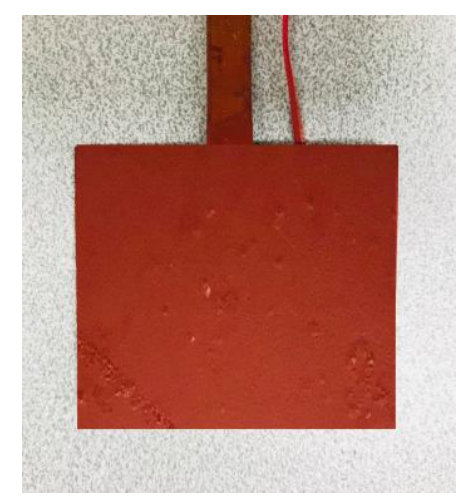

Figure 19 Coated sample with $\mu$ LPR sensor

After applying the primer, the $\mu$-strain gauge sensor was installed on top of the sample as shown in Figure 20. This requires careful placing, sticking and soldering of its terminal with wires. The coated sample with the attached $\mu$-strain gauge sensor is shown in Figure 21. 


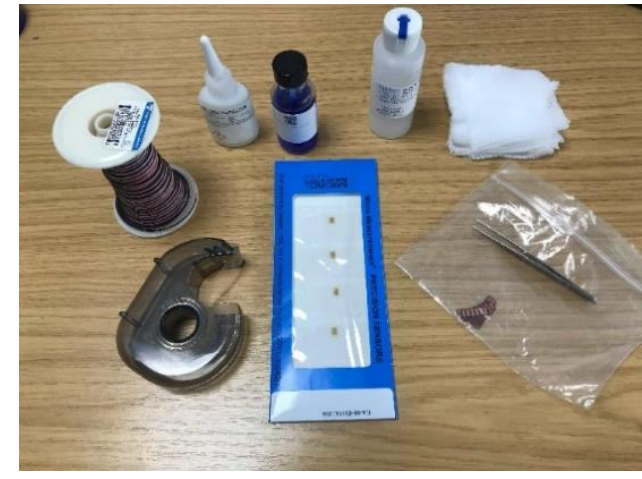

Figure 20 Accessories for installation of $\mu$-strain gauge sensor

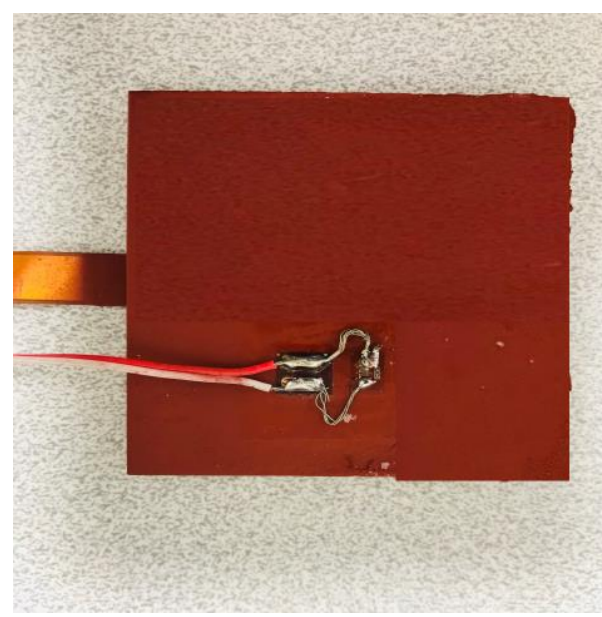

Figure 21 Sample with $\mu$-strain gauge sensor

A series of experiments were performed in an environmental chamber which provided harsh environmental conditions. The sample was immersed in $10 \%$ salt solution except where the $\mu$-strain gauge sensor was attached in order to avoid short circuit problem. The sample along with sensors inside the environmental chamber is shown in Figure 22.
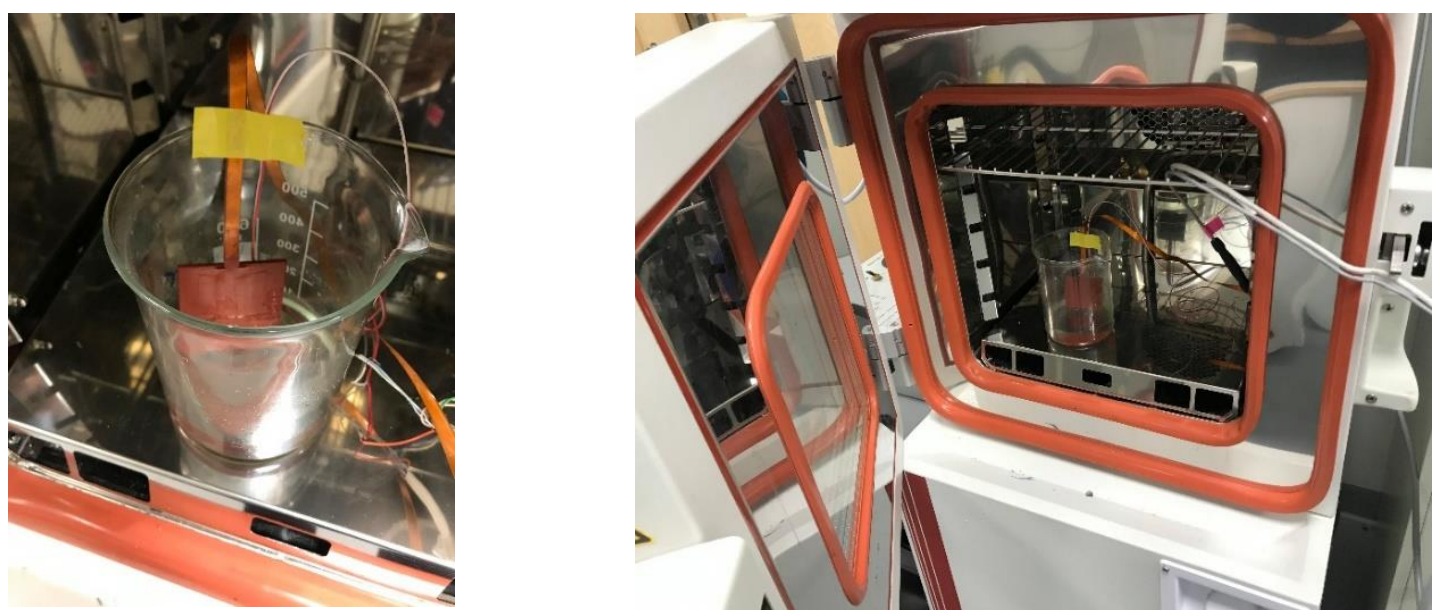

Figure 22 Sample inside environmental chamber 


\section{Results and discussion}

Various experiments have been performed to validate the response of the proposed RTU system with $\mu \mathrm{LPR}$ and $\mu$-strain gauge sensors. The stress/strain behaviour was observed by providing small and large temperature gradients. In the first experiment, the sample was kept in solution to detect the corrosion reaction under the coating and the development of stresses was observed by providing a large temperature gradient. The sensor measurements were recorded continually with a 30s interval for strain monitoring and $1 \mathrm{~min}$ interval for monitoring any corrosion development. The experiment was started at a temperature of $313.15 \pm 2 \mathrm{~K}$ and after $3 \mathrm{hrs}$ the temperature was decreased to $283.15 \pm 2 \mathrm{~K}$. It was observed that the tensile strain/stress continued to increase as the temperature was raised from room temperature to $313.15 \pm 2 \mathrm{~K}$, as shown in Figure 23 . The initial value of strain recorded was considered as being an offset that was produced during the application of the sensor. This offset value was deducted from subsequent strain measurements in order that the initial measurement started from zero on the graph. The value of tensile strain started from $0 \mu \varepsilon$ and reached a maximum of around $1200 \mu \varepsilon$. When a large negative temperature gradient of $\Delta T=-$ $30 \mathrm{~K}$ was applied, the $\mu$-strain gauge sensor experienced compressive strain and a very sharp change in the behaviour of sensor measurements were observed from around $1200 \mu \varepsilon$ to $-200 \mu \varepsilon$. The $\mu \mathrm{LPR}$ sensor under the coating detects the corrosion reaction and. In the absence of corrosion, it recorded a maximum linear polarisation resistance of $5 \mathrm{xe}^{6}$. The sensor face connected to the substrate acts as the working electrode. The resistance decreases in the presence of corrosion in the substrate. The results presented in Figure 24 show the detection of the corrosion reaction as the sample was dipped into the salt solution. The detection of corrosion also confirms the weak coating barrier due to the presence of micro-cracks that allowed the salt solution to penetrate.

In the second experiment, a negative temperature gradient was applied, although the gradient was low when compared to the previous experiment. The strain measurements recorded during the second experiment are presented in Figure 25. Initially, the environmental chamber was set at room temperature for $30 \mathrm{mins}$ and there was no significant change in strain as measured by the $\mu$-strain gauge. When a negative temperature gradient of $\Delta T=-18 \mathrm{~K}$ was applied, a compressive strain developed, which decreased from $0 \mu \varepsilon$ to $-900 \mu \varepsilon$. In addition, a sample was kept out of the salt solution for the initial $\mathbf{3 0}$ mins in order to observe if any corrosion occurs under the coating in the absence of the salt solution. No significant corrosion was detected in these first 30 mins, as shown in Figure 26. Corrosion on the substrate under the coating did start as the sample was dipped into solution, as shown by the decrease in linear polarisation resistance.

In the third experiment, the temperature gradient was applied in both the positive and negative directions including a small variation in temperature gradient, as shown in Figure 27. The initial temperature of $292.35 \pm 2 \mathrm{~K}$ produced a compressive strain and a small temperature gradient of $\Delta \mathrm{T}=$ $-3.3 \mathrm{~K}$ can also be observed, which is resulted in a slight increase in compressive strain. The temperature was increased after $2.8 \mathrm{hrs}$ which resulted in tensile strain and the value of strain increased. The corrosion showed interesting behaviour which can be linked with the development of stresses within the coating, based on literature $[2,21]$. During the negative change in temperature during the first $2.8 \mathrm{hrs}$ of the experiment, the corrosion detected was low when compared to that observed after $2.8 \mathrm{hrs}$, which had the highest corrosion rate throughout as shown in Figure 28. It was concluded that the negative temperature during the cycle before $2.8 \mathrm{hrs}$ resulted in compressive 
stresses within the coating which reduces the diffusion of the salt solution. The positive temperature gradient of $\Delta T=8 \mathrm{~K}$, however, resulted in tensile strain which expanded the micro-cracks within the coating and allowed greater diffusion of salt solution which resulted in a high corrosion rate.

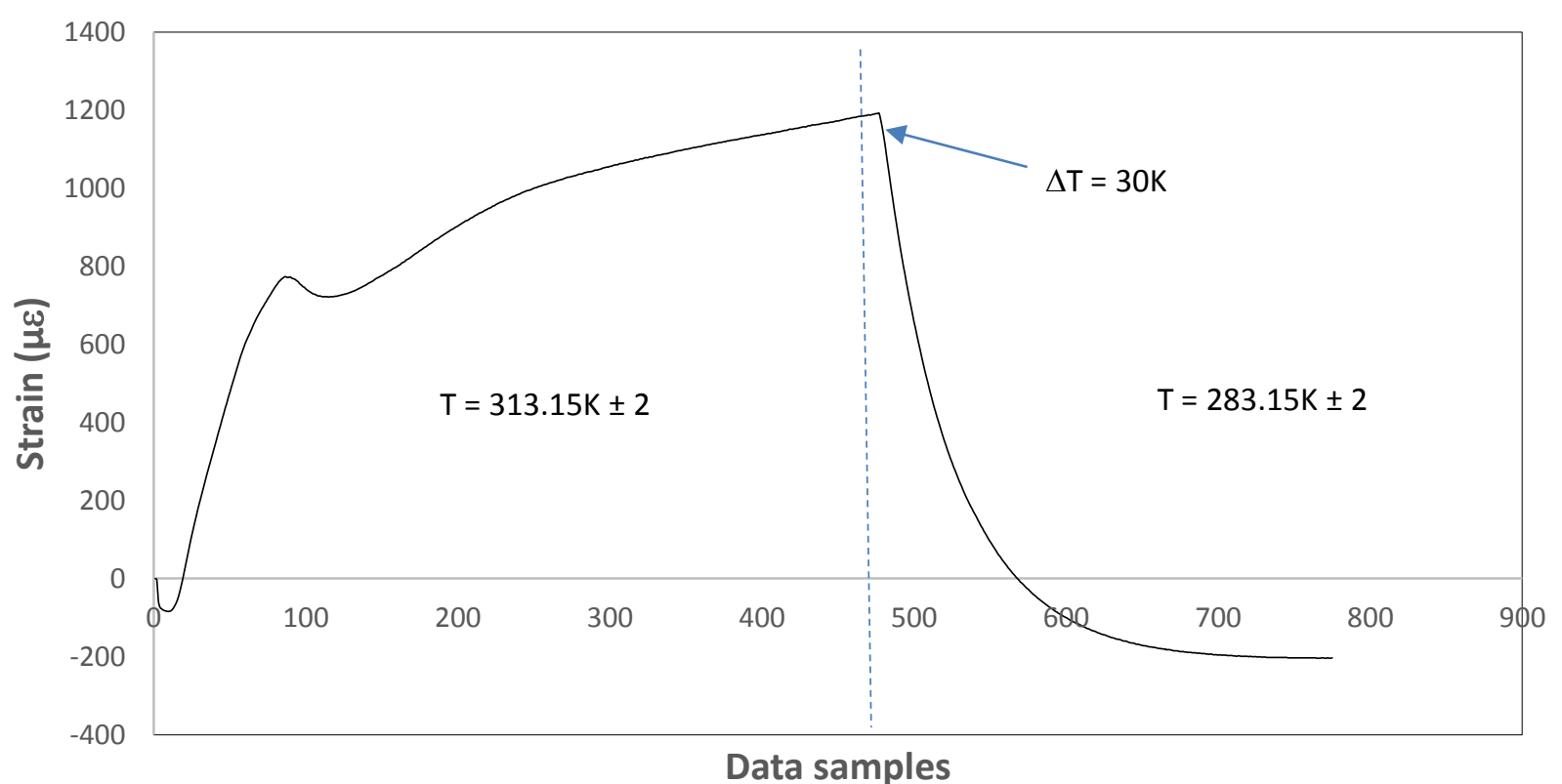

Figure 23 The measurements of $\mu$-strain gauge sensor during $1^{\text {st }}$ experiment 


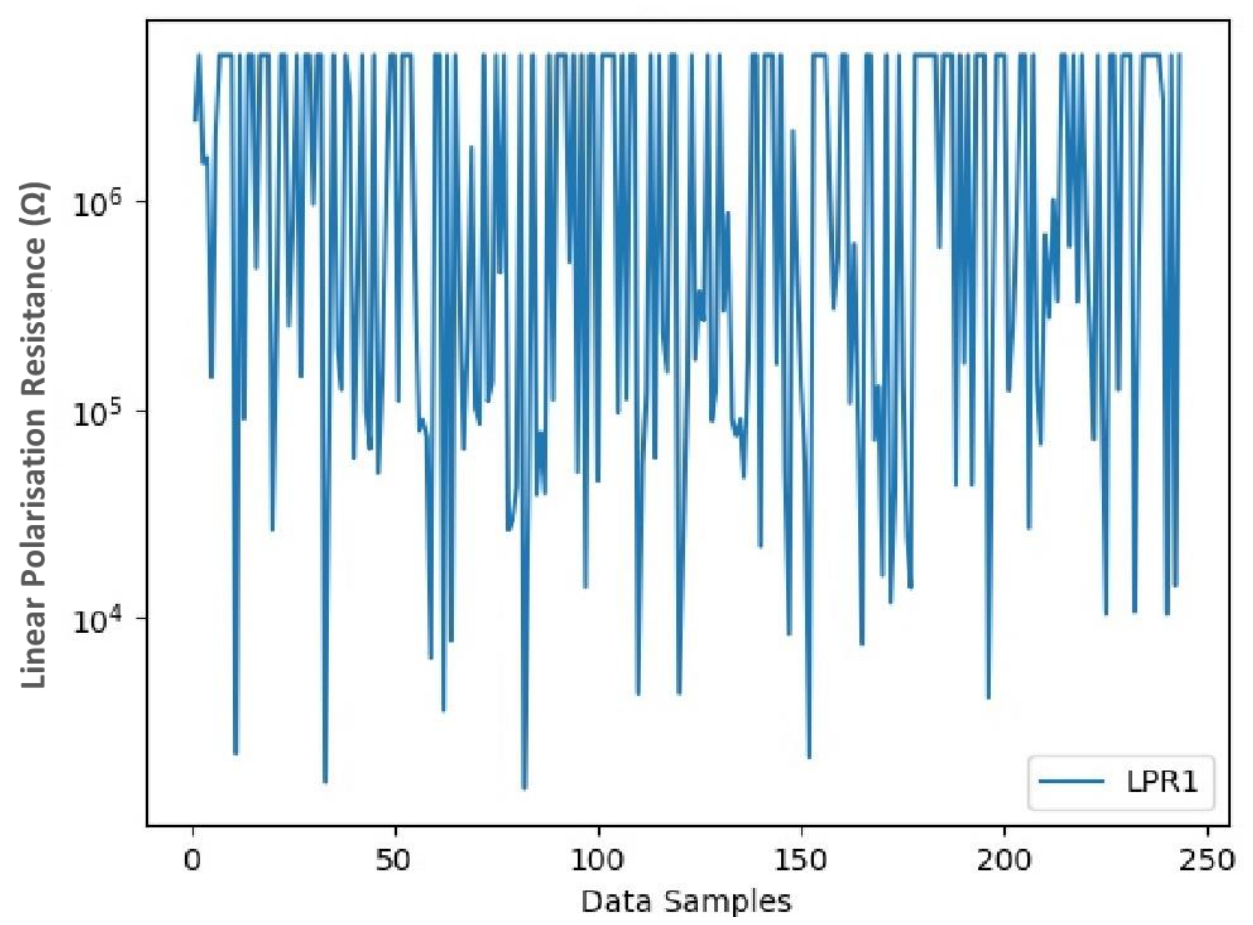

Figure 24 The measurements of $\mu$ LPR sensor during $1^{\text {st }}$ experiment

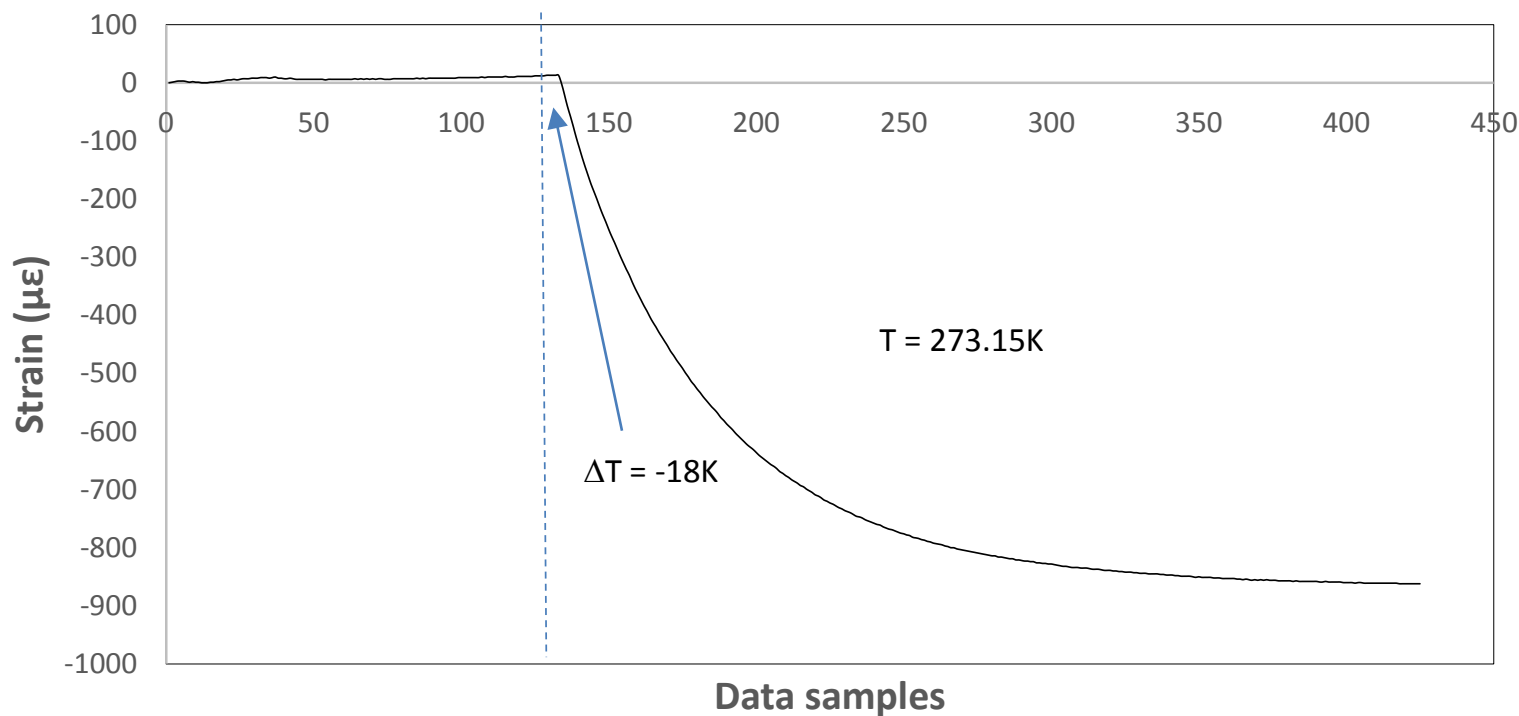

Figure 25 The measurements of $\mu$-strain gauge sensor during $2^{\text {nd }}$ experiment 


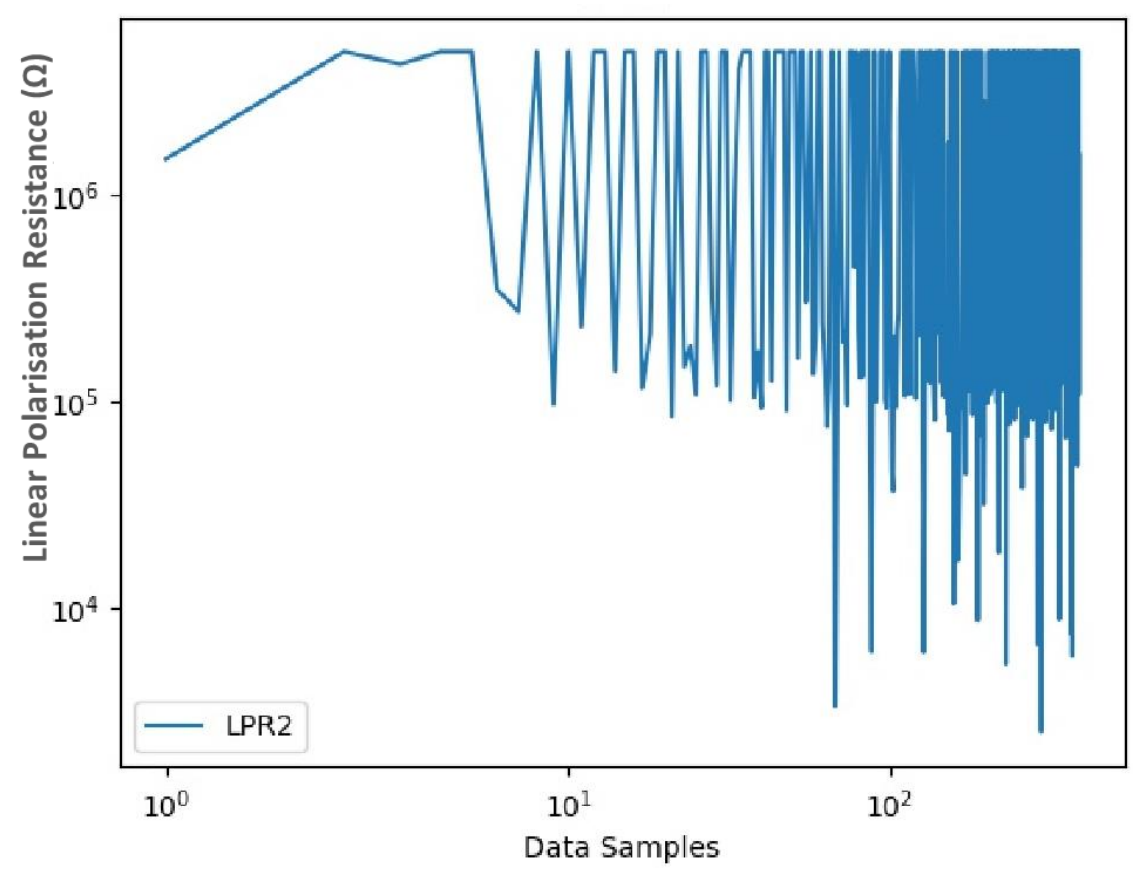

Figure 26 The measurements of $\mu$ LPR sensor during $2^{\text {nd }}$ experiment

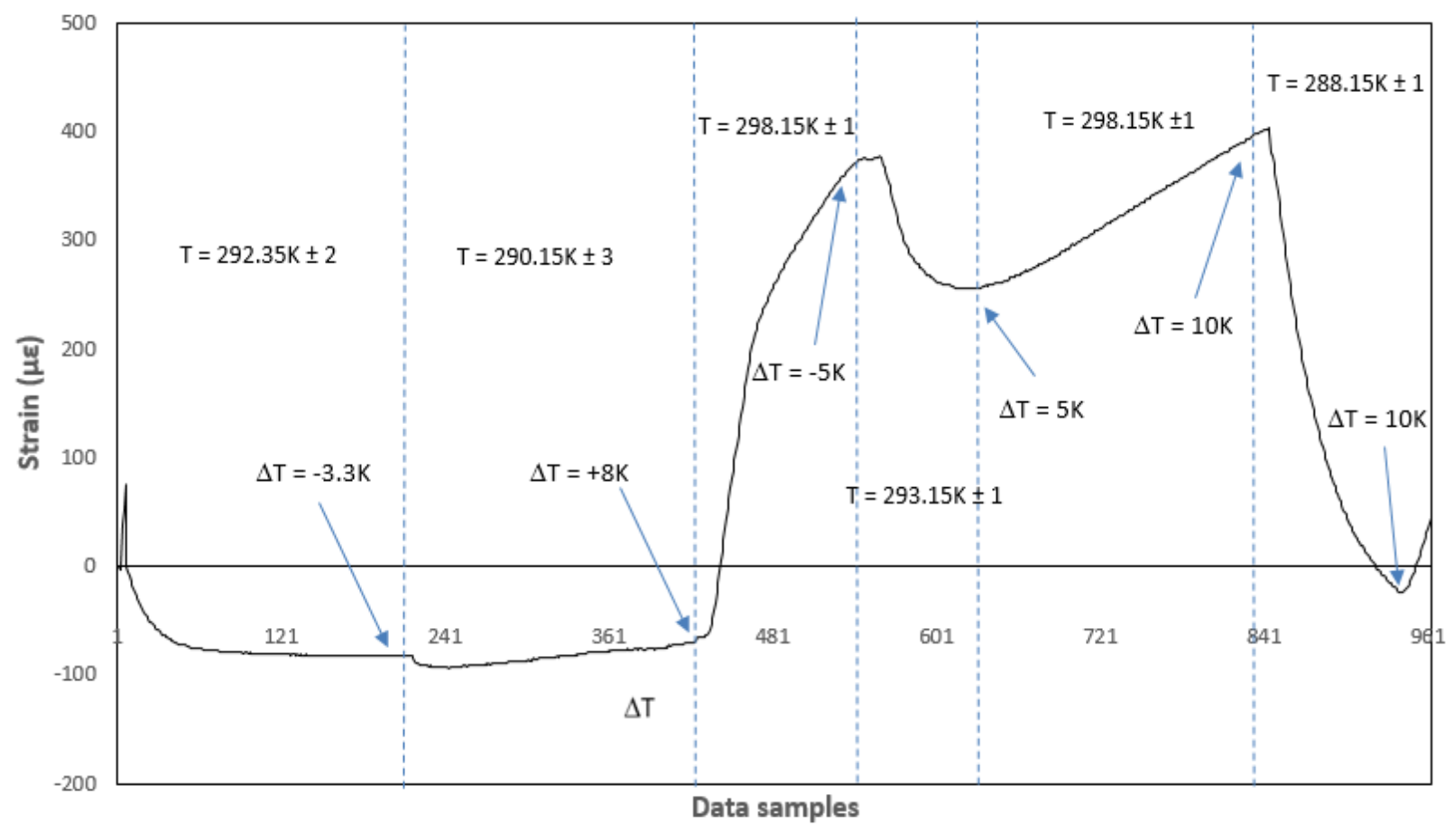

Figure 27 The measurements of $\mu$-strain gauge sensor during $3^{\text {rd }}$ experiment 


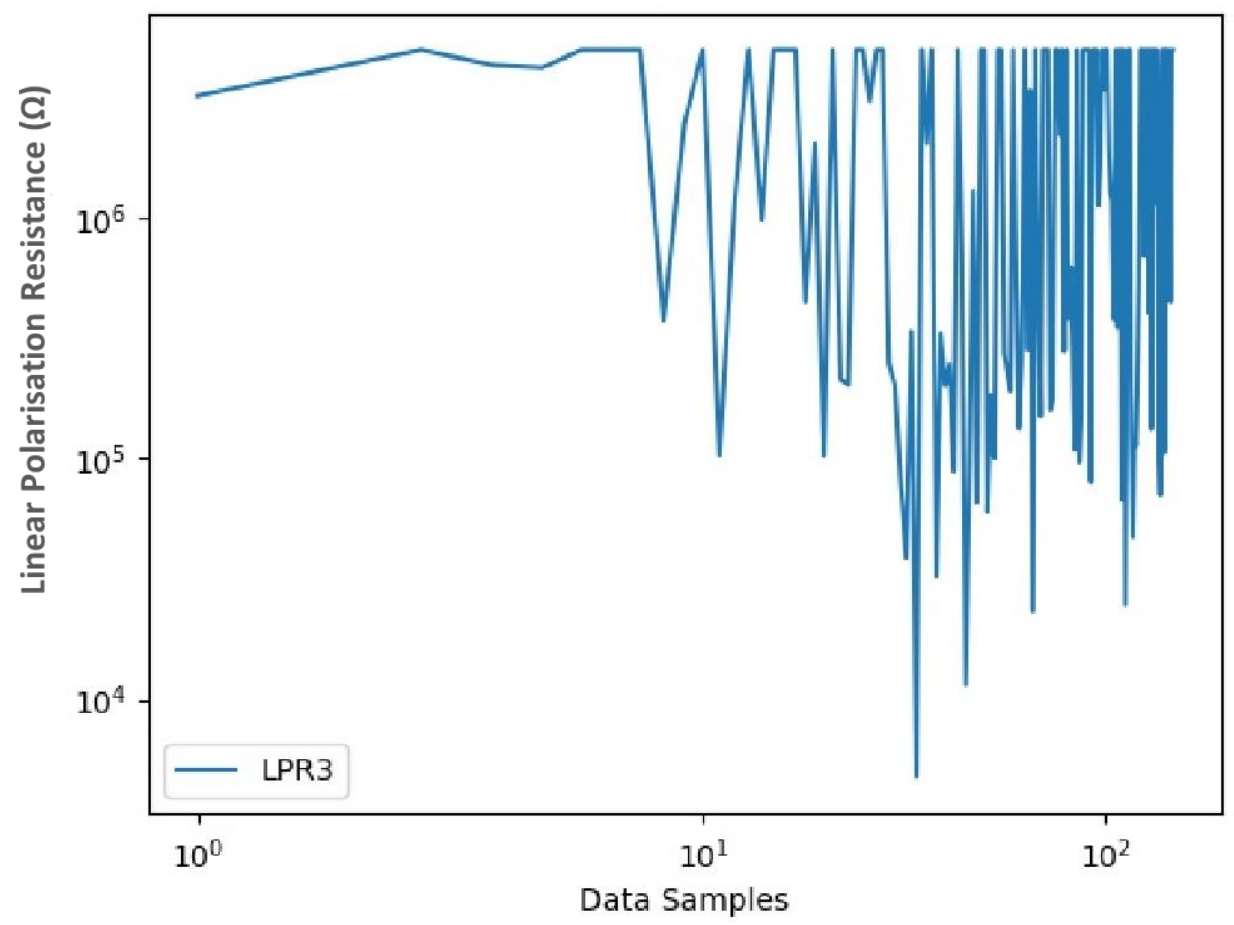

Figure 28 The measurements of $\mu \mathrm{LPR}$ sensor during $3^{\text {rd }}$ experiment

Several layers of coating were applied on substrate which seems to be protecting overall area. The $\mu$-LPR has detected corrosion under the coating in all three samples due to the penetration of salt solution. It can be considered that even after applying the coating on large vehicles which are stationed outside in uncontrolled atmospheric conditions, It is possible that the accumulation of salt particles from atmosphere and rain water can result into salt solution and penetrate into the interface of coating-substrate system to cause electrochemical reactions and keeps damaging the structure.

Large vehicles exposed to temperature gradient when they are often moved from the shed (in Museum) to outside environment for various activities. The temperature gradient responsible in developing residual stresses controls the diffusion of salt particles. The current findings has shown the potential of using strain gauge to observe the development of residual stresses subjected to small or large temperature gradients. There are many holistic and multidisciplinary coating failure and corrosion models can be found in literature, however the real time information regarding mechanical and electrochemical input parameters to the models is still grey area. The inspection and assessment of corrosion damage beneath the coating is also very challenging to schedule the maintenance especially for the structures operating at remote locations. The existence of microcracks within the coating is not visible during visual inspection. The results analysis from proposed SMS has shown that the combination of $\mu$-LPR and $\mu$-strain gauges provides an effective solution for real time monitoring of mechanical and electrochemical parameters. 


\section{Conclusion}

The experimental investigations has shown how invisible and minor coating defects contineously detoraite the structure in the presence of salt and water. The salinity in atmpsohere and rain are the primary sources of salt and water for sturcutres at remote locations. The comibantion of $\mu$-LPR and $\mu$-strain gauge sensors provides an effective solution to contineously monitor the phenomena leading to cororison under the coating. The current research has provided very comprehensive experimental investigations and development system of a structural health monitoring system. This system focuses on corrosion detection due to diffusion of corrosive particles and the development of stresses that result in various forms of coating failure. Future work requires comprehensive experimental work to create the benchmark for the development of stresses leading to coating failure. The threshold levels of failure depend on the properties of the material of structures, operating conditions and geometrical configurations. Therefore, experimental methods need to be designed in such a way that all necessary factors for efficient condition-based maintenance are considered.

\section{References}

1. 2016; Available from: https://www.nace.org/Newsroom/NACE-News/Study-Sets-CourseToward-Corrosion-Management-Practices-to-Increase-Safety,-Decrease-\$2-5-Trillion-GlobalCost-of-Corrosion/.

2. Nazir, M., et al., Modeling the effect of residual and diffusion-induced stresses on corrosion at the interface of coating and substrate. Corrosion, 2015. 72(4): p. 500-517.

3. Nazir, M., Z.A. Khan, and K. Stokes, Optimisation of interface roughness and coating thickness to maximise coating-substrate adhesion-a failure prediction and reliability assessment modelling. Journal of Adhesion Science and Technology, 2015. 29(14): p. 14151445.

4. Latif, J., et al., Condition monitoring and predictive modelling of coating delamination applied to remote stationary and mobile assets. Structural Health Monitoring, 2018.

5. Nazir, M., Z.A. Khan, and K. Stokes, A holistic mathematical modelling and simulation for cathodic delamination mechanism-a novel and an efficient approach. Journal of Adhesion Science and Technology, 2015. 29(22): p. 2475-2513.

6. Latif, J., et al., Life assessment prognostic modelling for multi-layered coating systems using a multidisciplinary approach. Materials Science and Technology, 2018. 34(6): p. 664-678.

7. Nazir, M.H. and Z. Khan, Maximising the interfacial toughness of thin coatings and substrate through optimisation of defined parameters. International Journal of Computational Methods and Experimental Measurements, 2015. 3(4): p. 316-328.

8. Nazir, M., Z. Khan, and K. Stokes, Modelling of metal-coating delamination incorporating variable environmental parameters. Journal of Adhesion Science and Technology, 2015. 29(5): p. 392-423.

9. Nazir, M.H., Z.A. Khan, and A. Saeed, A Novel Non-Destructive Sensing Technology for On-Site Corrosion Failure Evaluation of Coatings. IEEE Access, 2018. 6: p. 1042-1054.

10. Khan, Z.A., et al., Predictive and prognostic modelling and simulation of coatings subject to corrosion and mechanical failures. Materials Characterisation, 2018. 6(3): p. 487-498.

11. Nazir, M., et al., A predictive model for life assessment of automotive exhaust mufflers subject to internal corrosion failure due to exhaust gas condensation. Engineering Failure Analysis, 2016. 63: p. 43-60. 
12. Nazir, M. and Z.A. Khan, A review of theoretical analysis techniques for cracking and corrosive degradation of film-substrate systems. Engineering Failure Analysis, 2017. 72: p. 80-113.

13. Brown, D.W., et al. Linear Polarization Resistance Sensor Using the Structure as a Working Electrode. in Proceedings of the Second European Conference of the Prognostics and Health Management Society, Nantes, France. 2014.

14. Harris, S., M. Mishon, and M. Hebbron. Corrosion sensors to reduce aircraft maintenance. in Rto avt-144 workshop on enhanced aircraft platform availability through advanced maintenance concepts and technologies. Vilnius, Lithuania. 2006.

15. Nazir, M., A. Saeed, and Z.A. Khan, Electrochemical corrosion failure analysis of large complex engineering structures by using micro-LPR sensors. Sensors and Actuators B: Chemical, 2018. 268: p. 232-244.

16. Rossini, N., et al., Methods of measuring residual stresses in components. Materials \& Design, 2012. 35: p. 572-588.

17. Khan, Z.A., et al., Sensor based corrosion condition monitoring of coating substrate system informed by fracture mechanics, electrochemistry and heat transfer concepts, in Department of Defense - Allied Nations Technical Corrosion Conference. 2017: Birmingham, AL, USA.

18. Revie, R.W., Corrosion and corrosion control: an introduction to corrosion science and engineering. 2008: John Wiley \& Sons.

19. ASTM G59, in Standard Test Method for Conducting Potentiodynamic Polarization Resistance Measurements. 97(2014).

20. Aluminium Alloys - Aluminium 6082 Properties, Fabrication and Applications. Available from: https://www.azom.com/article.aspx?ArticlelD=2813.

21. Nazir, M., Z.A. Khan, and K. Stokes, Analysing the coupled effects of compressive and diffusion induced stresses on the nucleation and propagation of circular coating blisters in the presence of micro-cracks. Engineering Failure Analysis, 2016. 70: p. 1-15. 